\title{
Effects of disulfide bond reducing agents on sperm chromatin structural integrity and developmental competence of in vitro matured oocytes after intracytoplasmic sperm injection in pigs
}

\author{
Wen-Min Cheng ${ }^{1,2}$, Lei $\mathrm{An}^{1}$, Zhong-Hong Wu ${ }^{1}$, Yu-Bo Zhu ${ }^{1}$, Jing-Hao Liu ${ }^{1}$, Hong-Mei Gao ${ }^{1}$,

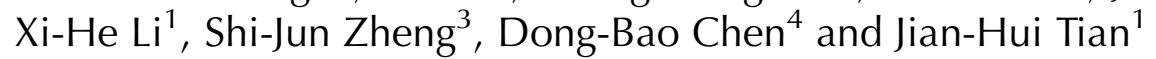 \\ ${ }^{1}$ Key Laboratory of Animal Genetics and Breeding of the Ministry of Agriculture, College of Animal Sciences and \\ Technology, China Agricultural University, Beijing 100094, People's Republic of China, ${ }^{2}$ College of Animal Sciences \\ and Technology, Yunnan Agricultural University, Kunming 650201, People's Republic of China, ${ }^{3}$ College of Veterinary \\ Medicine, China Agricultural University, Beijing 100094, People's Republic of China and ${ }^{4}$ Department of Obstetrics \\ and Gynecology, University of California Irvine, 101 Theory, Suite 200, Irvine, California 92697, USA
}

Correspondence should be addressed to J-H Tian; Email: tianjh@cau.edu.cn

W-M Cheng and L An contributed equally to this work

\begin{abstract}
We recently reported that electrical activation followed by secondary chemical activation greatly enhanced the developmental competence of in vitro matured porcine oocytes fertilized by intracytoplasmic sperm injection (ICSI). We hypothesized that sperm treatment with disulfide bond reducing agents will enhance the development competence of porcine embryos produced by this ICSI procedure. We examined the effects of glutathione (GSH), dithiothreitol (DTT), GSH or DTT in combination with heparin on sperm DNA structure, paternal chromosomal integrity, pronuclear formation, and developmental competence of in vitro matured porcine oocytes after ICSI. Acridine orange staining and flow cytometry based sperm chromatin structure assay were used to determine sperm DNA integrity by calculating the cells outside the main population (COMP $\alpha \mathrm{T})$. No differences were observed in COMP $\alpha \mathrm{T}$ values among GSH-treated and control groups. COMP $\alpha$ T values in GSH-treated groups were significantly lower than that in DTT-treated groups. Following ICSI, GSH treatments did not significantly alter paternal chromosomal integrity. Paternal chromosomal integrity in sperm treated with DTT plus or minus heparin was also the lowest among all groups. GSH-treated sperm yielded the highest rates of normal fertilization and blastocyst formation, which were significantly higher than that of control and DTT-treated groups. The majority of blastocysts derived from control and GSH-treated spermatozoa were diploid, whereas blastocysts derived from DTT-treated spermatozoa were haploid. In conclusion, sperm treatment with GSH enhanced the developmental capacity of porcine embryos produced by our optimized ICSI procedure.

Reproduction (2009) 137 633-643
\end{abstract}

\section{Introduction}

Intracytoplasmic sperm injection (ICSI) describes a procedure in which a single sperm is injected directly into the cytoplasm of an oocyte to facilitate fertilization. Clinically, this assisted fertilization approach is presently used for treating male infertility in men with an extremely low number of and/or immotile spermatozoa (Tesarik 1996). ICSI is also a powerful means of producing transgenic animals by sperm-mediated gene transfer (Perry et al. 1999). In comparison to the commonly used pronuclear microinjection procedure, ICSI offers higher integration frequency of exogenous genes into the genome of the founder offsprings. Moreover, ICSI makes it possible to introduce large DNA molecules such as yeast or mammalian artificial chromosomes $(>250$ kilobases in size) into an oocyte without the threat of 'shearing' of the DNA molecule (Perry et al. 1999, Martin 2000). Thus, ICSI is considered to be a very useful technique for generating transgenic pigs (Lai et al. 2001).

The application of ICSI in pigs has not been as simple as originally thought (Nakai et al. 2003). It has been reported that fertilization rate by ICSI is low in pigs, e.g., $\sim 20 \%$ on average (Kolbe \& Holtz 2000, Martin 2000, Nakai et al. 2003, Watanabe \& Fukui 2006, Katayama et al. 2007). Although the causes of the low success rate in porcine ICSI are uncertain, incomplete oocyte activation (Kolbe \& Holtz 1999, 2000) and failure of male pronucleus formation have been suspected as the two pivotal factors for causing the low developmental capacity of ICSI-fertilized porcine embryos 
(Thibault 1972, Kren et al. 2003, Lee et al. 2003). More recently, we reported that electrical activation followed by a secondary chemical activation with 6-dimethylaminopurine (6-DMAP) could significantly enhance the activation rate of porcine oocytes (Tian et al. 2006). However, the sperm treatment regimen for maximizing the developmental capacity of ICSIfertilized porcine embryos has yet to be determined.

During normal fertilization, in vivo sperm nuclear decondensation requires two major steps, i.e., reduction of $\mathrm{S}-\mathrm{S}$ to $\mathrm{S}-\mathrm{H}$ in protamines followed by replacement of protamines with histones (Zirkin et al. 1989). Reduction of $\mathrm{S}-\mathrm{S}$ to $\mathrm{S}-\mathrm{H}$ depends on the presence of the free thiol glutathione (GSH; Perreault et al. 1988, Zirkin et al. 1989). Treatment with dithiothreitol (DTT) to reduce disulfide bonds in protamines, prior to ICSI, enhanced spermatozoa chromosomal decondensation and subsequent formation of a male pronucleus in bovine zygotes (Rho et al. 1998). However, DTT-treated sperm did not improve the developmental competence of porcine embryos (Yong et al. 2005, Nakai et al. 2006). By contrast, when combined with detergents, DTT was detrimental to embryonic development as it breaks paternal chromosomes in the zygote (Szczygiel \& Ward 2002). Thus, new methods for sperm preparation are needed for improving the developmental competence of ICSI-fertilized porcine embryos. As an endogenous disulfide bond reducer, reduced GSH is present in the cytoplasm of oocytes, which could protect oocytes against oxidative stress (Perreault et al. 1984, 1988). This knowledge has directed the use of GSH in inducing in vitro sperm decondensation in human (Reyes et al. 2005), bovine (Delgado et al. 2001), and hamster zygotes (Francisco et al. 1998). To the best of our knowledge, GSH has not yet been tested for sperm preparation to improve the developmental competence of ICSI-fertilized porcine embryos.

Previous studies have shown that heparin can decondense sperm nuclei in vivo (Lalich et al. 1989, Montag et al. 1992). Heparin and other polyanions induce sperm nuclear decondensation by depletion of protamines from the chromatin (Jager et al. 1990). In the presence of a disulphide bond reducing agent, relatively low concentrations of heparin induced rapid nuclear decondensation in bovine, mouse, and human sperm (Jager et al. 1990). Other polyanions also can decondense sperm nuclei, but with lesser efficacy than heparin (Jager et al. 1990). Romanato et al. (2003) reported that the ability of heparin to decondense human sperm in vitro is related to its structural characteristics rather than its nature of being a polyanion. Whether heparin in combination with other disulphide bond reducing agents (i.e., GSH, DTT) enhances porcine sperm nuclear decondensation is presently unknown. Based on an oocyte activation protocol optimized in our laboratory (Tian et al. 2006), the present series of experiments were performed to study: 1 ) the effects of GSH, DTT, GSH or
DTT in combination with heparin on porcine sperm chromatin structure; 2) the effects of these sperm treatments on the paternal chromatin structure; and 3) the developmental competence of the porcine ICSIfertilized embryos using these treated sperm in vitro.

\section{Results \\ Effects of disulfide reducing chemical treatments on sperm chromatin structure}

After treatment with different disulfide reducing chemicals, spermatozoa were stained with $\mathrm{AO}$ and evaluated for chromatin structure by flow cytometry. The results were expressed as the mean $(\mathrm{X} \alpha \mathrm{T})$ and $\mathrm{SD}$ (SD $\alpha \mathrm{T})$ of $\alpha \mathrm{T}$ distribution, the percentage of cells with high $\alpha \mathrm{T}$ values referred to as cells outside the main population (COMP $\alpha \mathrm{T}$ ), and the mean (X Green) and SD (SD Green) of the green fluorescence intensity distribution. The results are summarized in Fig. 1 and Table 1.

As listed in Table 1, the mean values of $\alpha \mathrm{T}$ were significantly different among all groups $(P<0.05)$. The $\alpha \mathrm{T}$ values of treatments with DTT in the absence $(597.7 \pm 7.7)$ or presence $(578.3 \pm 9.0)$ of heparin significantly increased compared with those of control $(250.9 \pm 2.3)$ and treatments with GSH in the absence $(229.1 \pm 5.4)$ and presence $(263.2 \pm 4.4)$ of heparin. No differences $(P>0.05)$ were observed in the COMP $\alpha \mathrm{T}$ values among control and GSH treatments with or without heparin. The values of COMP $\alpha \mathrm{T}$ markedly increased as mean values of $\alpha \mathrm{T}$ increased. The values of COMP $\alpha \mathrm{T}$ in DTT treatments without $(29.5 \pm 0.6)$ and with $(36.2 \pm 10.9)$ heparin were higher $(P<0.05)$ than those in untreated control $(3.7 \pm 0.6)$ and GSH without $(7.0 \pm 0.8)$ or with $(12.2 \pm 2.4)$ heparin. As the COMP $\alpha \mathrm{T}$ value increases with sperm chromatin damage, DTT treatments apparently caused a significant increase in the sperm DNA damage. The mean values of the green fluorescence intensity distributions were lower $(P<0.05)$ in control $(481.7 \pm 25.5)$, GSH $(470.8 \pm 5.0)$, and GSH + heparin (537.8 \pm 5.4 ) groups than those in DTT $(645.9 \pm 8.5)$ and DTT + heparin $(849.8 \pm 78.1)$ groups. No significant differences were observed in the mean values of the green fluorescence intensity distribution among control and GSH without or with heparin treatments $(P<0.05)$.

\section{Chromosomal integrity in ICSI-fertilized zygotes}

The chromosomal integrity of the ICSI-fertilized zygotes before the first cleavage was examined and data are summarized in Table 2. Treatment with DTT minus $(8.92 \%)$ or plus $(5 \%)$ heparin did not cause significant increases in the chromosomal damage of the paternal chromosomes with minor or multiple aberrations compared with that of control $(1.72 \%)$ and treatments with GSH in the absence $(1.75 \%)$ and presence $(5 \%)$ of heparin $(P>0.05)$. 

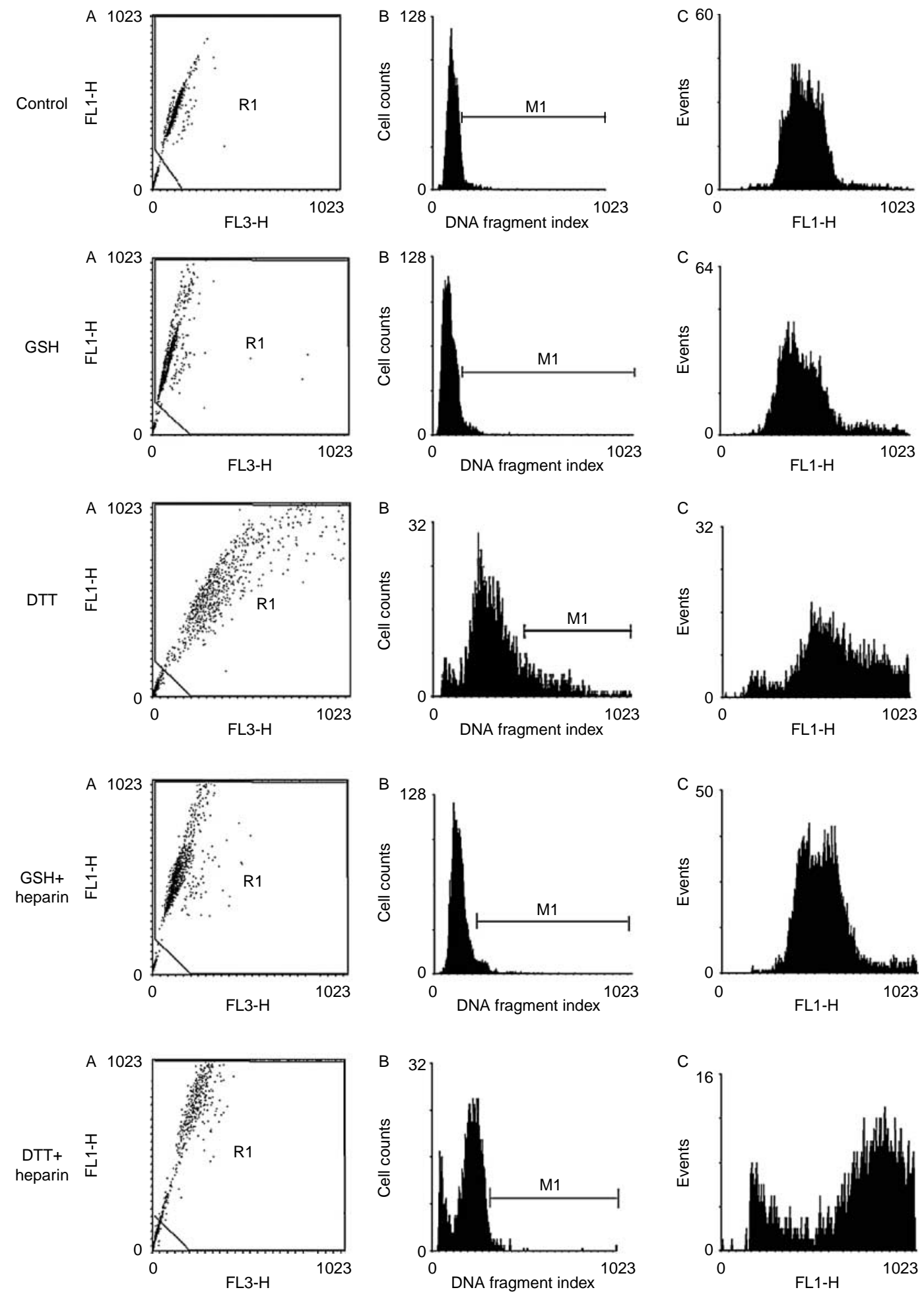

Figure 1 Typical histogram of the AO fluorescence patterns from porcine spermatozoa treated with different disulfide bond reducing agents. A total of 10000 events were measured and accumulated in the cytogram, each dot representing one spermatozoon. (A) Representative green (FL1-H) versus red (FL3-H) fluorescence bivariate histogram; (B) DNA fragment index (ratio of red to red + green fluorescence) intensity; (C) green fluorescence distributions. 
Table 1 Effects of different disulfide bond reducing agents on porcine sperm chromatin structure assay by flow cytometry.

\begin{tabular}{|c|c|c|c|c|c|}
\hline FCM SCSA & Control & GSH & DTT & GSH + heparin & DTT + heparin \\
\hline $\mathrm{X} \alpha \mathrm{T}$ & $250.9 \pm 2.3^{+}$ & $229.1 \pm 5.4^{*}$ & $597.7 \pm 7.7^{\| 1}$ & $263.2 \pm 4.4^{\ddagger}$ & $578.3 \pm 9.0^{\S}$ \\
\hline $\mathrm{SD} \alpha \mathrm{T}$ & $84.3 \pm 11.0 *$ & $85.1 \pm 9.3^{*}$ & $176.5 \pm 2.4^{+}$ & $88.2 \pm 8.7^{*}$ & $180.1 \pm 7.0^{+}$ \\
\hline COMP $\alpha \top$ & $3.7 \pm 0.6^{*}$ & $7.0 \pm 0.8^{*}$ & $29.5 \pm 0.6^{+}$ & $12.2 \pm 2.4^{*}$ & $36.2 \pm 10.9^{+}$ \\
\hline X Green & $481.7 \pm 25.5^{*}$ & $470.8 \pm 5.0^{*}$ & $645.9 \pm 8.5^{+}$ & $537.8 \pm 5.4^{*}$ & $849.8 \pm 78.1^{\neq}$ \\
\hline SD Green & $108.8 \pm 14.4^{*}$ & $143.5 \pm 6.1^{\dagger}$ & $198.7 \pm 4.3^{\ddagger}$ & $133.3 \pm 5.0^{\dagger}$ & $194.8 \pm 12.1^{\ddagger}$ \\
\hline
\end{tabular}

FCM, flow cytometric method; SCSA, sperm chromatin structure assay; $\alpha \mathrm{T}$, ratio of red to total (red plus green) fluorescence intensity; $\mathrm{X} \alpha \mathrm{T}$, the mean value of $\alpha \mathrm{T}$ distribution; SD $\alpha \mathrm{T}$, the SD of $\alpha \mathrm{T}$ distribution; COMP $\alpha \mathrm{T}$, cells outside the main population; $\mathrm{X}$ Green, the mean value of the green fluorescence intensity; SD Green, the SD of the green fluorescence intensity. Values in the same row with different superscripts differ significantly $(P<0.05)$.

\section{Effects of sperm treatments with disulfide bond reducing chemicals on pronuclear formation and developmental competence of ICSI-fertilized oocytes}

As summarized in Table 3, there were statistical differences $(P<0.05)$ among the activation rates of ICSI-fertilized oocytes by using sperm treated with different chemicals. Interestingly, normal fertilization rates of oocytes fertilized by ICSI with GSH (62\%), GSH + heparin $(51.16 \%)$, and DTT + heparin- $(45.83 \%)$ treated sperm were significantly greater $(P<0.05)$ than those of the control (33.33\%) and DTT-treated (38.78\%) groups. There were no differences among GSH, $\mathrm{GSH}+$ heparin, and DTT + heparin-treated groups and between control and DTT-treated groups $(P>0.05)$.

The developmental competence of the ICSI-fertilized embryos derived from sperm treated with different disulfide bond reducing reagents is summarized in Table 4 . The cleavage rates did not differ among all groups $(P>0.05)$; however, the blastocyst formation rates were significantly different among groups $(P<0.05)$. GSH-treated sperm yielded the highest of blastocyst formation rate $(40.21 \%)$, which was significantly higher than that of control $(19.60 \%)$, DTT$(24.72 \%)$, and DTT + heparin- $(28 \%)$ treated groups $(P<0.05)$. The blastocyst formation rate in the GSH+ heparin-treated group was also higher than that of control group $(32.26 \%$ vs $19.60 \%, P<0.05)$. Sperm treated with DTT in the absence or presence of heparin did not cause significant changes in blastocyst formation rates in comparison with control $(P>0.05)$. In addition, the cell number $(54.05 \pm 4.37)$ of the blastocyst formed in the DTT+heparin group was higher $(P<0.05)$ than that in control $(44.71 \pm 6.25)$, GSH $(38.96 \pm 4.72)$, DTT $(42.32 \pm 4.59)$, and GSH+ heparin (38.92 \pm 7.82$)$ groups.

Finally, we examined the karyotype of ICSI blastocysts derived from sperm treated with different chemicals. As summarized in Table 5, there was a dramatically increased haploid blastocyst rate $(80 \%)$ in the DTTtreated group than in other treatment groups, in which $\sim 60 \%$ of the blastocysts formed were diploid $(P<0.05)$. No polyploid blastocysts were observed in any of the groups.

\section{Discussion}

In most mammals, sperm nuclear decondensation in vivo seems to be composed of two distinct stages: reduction of disulfide bonds in protamines and replacement of protamines with histones (Zirkin et al. 1989). Therefore, in vitro, spermatozoa are often treated with disulfide bond reducing agents such as DTT or GSH for facilitating its nuclear decondensation. DTT is a commonly used disulfide bond reducing reagent, and sperm treatment with DTT has been used to increase the success rate of bovine and porcine ICSI.

In this study, we have first investigated the effects of different disulfide bond reducing agents (DTT, GSH, and their combination) on porcine sperm DNA structure, parental chromosomal integrity, and developmental competence of porcine ICSI embryos derived from the treated sperm. The flow cytometry based sperm chromatin structure assay was used to measure sperm chromatin integrity. This assay exploits the metachromatic properties of acridine orange $(\mathrm{AO})$ to monitor the

Table 2 Chromosomal aberrations in porcine zygotes after injection with spermatozoon treated with different disulfide bond reducing chemicals.

\begin{tabular}{|c|c|c|c|c|c|}
\hline Treatment & $\begin{array}{l}\text { No. of metaphases } \\
\text { examined }\end{array}$ & $\begin{array}{l}\text { No. of normal } \\
\text { metaphases (\%) }\end{array}$ & $\begin{array}{c}\text { No. of metaphases } \\
\text { with minor } \\
\text { aberrations (\%) }\end{array}$ & $\begin{array}{l}\text { No. of metaphases } \\
\text { with multiple } \\
\text { aberrations }(\%)\end{array}$ & $\begin{array}{l}\text { Total no. of } \\
\text { metaphases with } \\
\text { aberrations }(\%)\end{array}$ \\
\hline Control & 58 & $57(98.28)^{*}$ & $0(0)^{*}$ & $1(1.72)^{*}$ & $1(1.72)^{*}$ \\
\hline GSH & 57 & $55(96.49)^{*}$ & $0(0)^{*}$ & $1(1.75)^{*}$ & $1(1.75)^{*}$ \\
\hline DTT & 56 & $51(91.07)^{*}$ & $3(5.36)^{*}$ & $2(3.57)^{*}$ & $5(8.92)^{*}$ \\
\hline $\mathrm{GSH}+$ heparin & 56 & $54(96.43)^{*}$ & $2(3.57)^{*}$ & $0(0)^{*}$ & $2(3.57)^{*}$ \\
\hline DTT+heparin & 60 & $57(95)^{*}$ & $2(3.33)^{*}$ & $1(1.67)^{*}$ & $3(5)^{*}$ \\
\hline
\end{tabular}

Values within a column with different superscripts differ significantly $(P<0.05)$. 
Table 3 Effects of different disulfide bond reducing chemicals for porcine spermatozoa on pronuclear formation of porcine intracytoplasmic sperm injection embryos.

\begin{tabular}{|c|c|c|c|c|c|}
\hline \multirow[b]{2}{*}{ Nucleus morphology } & \multicolumn{5}{|l|}{ Treatment } \\
\hline & Control & GSH & DTT & GSH+heparin & DTT+heparin \\
\hline No. of oocytes examined & 48 & 50 & 49 & 43 & 48 \\
\hline Total no. $(\%)$ oocytes at $M \alpha$ stage & $12(25)^{*}$ & $6(12)^{*}$ & $12(24.49)^{*}$ & $8(18.60)^{*}$ & $5(10.42)^{*}$ \\
\hline No. $(\%)$ oocytes with $1 \mathrm{MPN}+1 \mathrm{~PB}$ & $8(16.67)^{*}$ & $6(12)^{*,+}$ & $10(20.41)^{*}$ & $6(13.95)^{*,+}$ & $2(4.17)^{+}$ \\
\hline No. $(\%)$ oocytes at $M \alpha$ stage $+1 P B$ & $4(8.33)^{*}$ & $0(0)^{*}$ & $2(4.08)^{*}$ & $2(4.65)^{*}$ & $3(6.25)^{*}$ \\
\hline Total no.(\%) oocytes activated & $36(75)^{*}$ & $44(88)^{*}$ & $37(75.51)^{*}$ & $35(81.40)^{*}$ & $43(89.58)^{*}$ \\
\hline No. $(\%)$ oocytes with $1 \mathrm{MPN}+1 \mathrm{FPN}+2 \mathrm{~PB}$ & $16(33.33)^{*}$ & $31(62)^{\dagger}$ & $19(38.78)^{*}$ & $22(51.16)^{*,+}$ & $22(45.83)^{*,+}$ \\
\hline No.(\%) oocytes with $1 \mathrm{MPN}+2 \mathrm{FPN}+1 \mathrm{~PB}$ & $0(0)^{*}$ & $0(0)^{*}$ & $1(2.04)^{*}$ & $0(0)^{*}$ & $0(0)^{*}$ \\
\hline No.(\%) oocytes with $2 \mathrm{FPN}+1 \mathrm{~PB}+$ sperm & $4(8.33)^{*}$ & $0(0)^{*}$ & $4(8.16)^{*}$ & $3(6.98)^{*}$ & $2(4.17)^{*}$ \\
\hline No. $(\%)$ oocytes with $1 \mathrm{FPN}+1 \mathrm{~PB}($ or $2 \mathrm{~PB})+$ sperm & $16(33.33)^{*}$ & $13(26)^{*}$ & $13(26.53)^{*}$ & $10(23.26)^{*}$ & $19(39.58)^{*}$ \\
\hline
\end{tabular}

Values in within a row with different superscripts are significantly different $(P<0.05)$. FPN, female pronuclear; PB, second polar body; MPN, male pronuclear; sperm, intact or condensed sperm head.

susceptibility of sperm chromatin DNA to acid-induced denaturation in situ (Evenson et al. 1980, 1991, Spanò \& Evenson 1993). The bivariate data can be conveniently expressed as function $\alpha \mathrm{T}(\alpha \mathrm{T}) \alpha \mathrm{T}$ is the ratio of red to total (red plus green) fluorescence intensity (Darzynckiewicz et al. 1975), which represents the amount of the denatured, single-stranded DNA over the total cellular DNA. Therefore, $\alpha$ T parameters can reflect the chromatin alterations associated with DNA nicks. When $\alpha \mathrm{T}$ value increases, the denatured or single strand DNA binding sites increases and so does the percentage of COMP $\alpha \mathrm{T}$ and the percentage of sperm with denatured DNA or damaged chromatin. DTT acts on disulfide bonds of sperm protamine and induces sperm chromatin decondensation (Rho et al. 1998, Tateno \& Kamiguchi 1999, Szczygiel \& Ward 2002, Yong et al. 2005). We found that the value of COMP $\alpha \mathrm{T}$ in DTT-treated sperm was higher than those of GSH-treated and control groups. These results indicate that DTT may increase the susceptibility of the sperm's DNA to other insults.

DTT treatment alone did not disrupt the integrity of paternal DNA (Ward et al. 2000). However, Szczygiel \& Ward (2002) reported that a combination of DTT and detergent causes paternal chromosomal damage in mice. Our current data suggest that without detergent, DTT alone caused more sperm DNA denatuaration than untreated control in pigs. The disulfide bonds (-SS-) in proteins make spermatozoa resistant to harsh physical treatments such as freeze-drying. A lack of the extensive -SS- cross-linking makes the nucleus of immature spermatozoa vulnerable to physical and chemical disruptions (Kaneko et al. 2003). DTT, which reduces $\mathrm{S}-\mathrm{S}$ bonds in the cysteine-rich protamines to $-\mathrm{SH}$, made mouse epididymal spermatozoa susceptible to damage from freeze-drying (Kaneko et al. 2003). Protamines are most likely to be responsible for the genetic (chromosomal) integrity of the sperm nucleus in the face of an artificial insult such as freeze-drying (Kaneko et al. 2003). Even very subtle alterations in the structural proteins of the nucleus are sufficient to deprive the sperm's DNA of the ability to support embryonic development (Ward et al. 2000). Sperm chromatin integrity is of critical importance during ICSI. The endogenous nucleases in mouse sperm can be activated by freeze-thawing, which in turn can cleave DNA in spermatozoa (Pérez-Crespo et al. 2008). The uterine content from females mated to vasectomized males and the seminal vesicle fluid have increased nuclease activities. When the epididymal sperm were exposed to these conditions, an increased incidence of sperm chromosome nicks and extremely severe DNA breakages were observed after ICSI. However, chromosomes were normal in sperm exposed to the uterine content from unmated females in estrus (Yamauchi et al. 2007). Based on these recent findings, we speculated that the harmful effects of DTT on porcine sperm observed in our present study very likely originated from the effects of sperm endonucleases. It is also possible that DTT destabilized the extensive -SS- crosslinking and altered the structural proteins of the nucleus

Table 4 Developmental competence of in vitro matured porcine oocytes fertilized by intracytoplasmic sperm injection using a spermatozoon treated with different disulfide bond reducing chemicals in vitro.

\begin{tabular}{lcccc}
\hline Treatment & Oocytes & Number of cleavages $(\%)$ & Number of blastocysts (\%) & Cell number of blastocysts \\
\hline Control & 71 & $60(84.31)^{*}$ & $14(19.60)^{\ddagger}$ & $44.71 \pm 6.25^{+}$ \\
GSH & 72 & $63(87.60)^{*}$ & $29(40.21)^{*}$ & $38.96 \pm 4.72^{+}$ \\
DTT & 89 & $82(92.13)^{*}$ & $22(24.72)^{+, \neq}$ & $42.32 \pm 4.59^{+}$ \\
GSH+heparin & 105 & $98(92.86)^{*}$ & $34(32.26)^{*,+}$ & $38.92 \pm 7.82^{+}$ \\
DTT+heparin & 75 & $70(93.33)^{*}$ & $21(28)^{+, \neq}$ & $54.05 \pm 4.37^{*}$ \\
\hline
\end{tabular}

Values within a column with different superscripts differ significantly $(P<0.05)$. 
Table 5 Karyotyping of porcine blastocysts derived from in vitro matured oocytes fertilized by intracytoplasmic sperm injection with spermatozoon treated with different disulfide bond reducing chemicals.

\begin{tabular}{lcccc}
\hline Treatment & $\begin{array}{l}\text { Blastocysts } \\
\text { (No.) }\end{array}$ & $\begin{array}{l}\text { Number of } \\
\text { haploid }(\%)\end{array}$ & $\begin{array}{l}\text { Number of } \\
\text { diploid }(\%)\end{array}$ & $\begin{array}{l}\text { Number of } \\
\text { polyploid } \\
(\%)\end{array}$ \\
\hline Control & 8 & $3(37.5)^{*}$ & $5(62.5)^{*}$ & 0 \\
GSH & 9 & $3(33.33)^{*}$ & $6(66.67)^{*}$ & 0 \\
DTT & 10 & $8(80)^{\dagger}$ & $2(20)^{+}$ & 0 \\
$\begin{array}{l}\text { GSH+ } \\
\text { heparin }\end{array}$ & 6 & $2(33.33)^{*}$ & $4(66.67)^{*}$ & 0 \\
$\begin{array}{l}\text { DTT+ } \\
\text { heparin }\end{array}$ & 10 & $4(40)^{*}$ & $6(60)^{*}$ & 0 \\
\hline
\end{tabular}

Values within a column with different superscripts differ significantly $(P<0.05)$.

in sperm, which makes them more susceptible to nuclease-dependent DNA damage (Sutovsky et al. 1997, Ward \& Ward 2004).

In the present study, we also used another disulfide bond reducer, GSH, to treat porcine spermatozoa. GSH, the most abundant low-molecular-weight non-protein thiol, exists in two forms: reduced GSH and GSH disulfide (GSSG). It has been clearly shown that GSH is a disulfide bond reducer present in the cytoplasm of oocytes (Perreault et al. 1984, 1988). Oocytes can synthesize GSH (tripeptide $\gamma$-glutamyl-cysteinyl-glycine; $\mathrm{GSH}$ ) during the first meiosis. Oocyte-derived GSH seems to assure the reduction of disulfide bonds in sperm nucleus. This, in turn, promotes nuclear decondensation for male pronucleus formation during fertilization (reviewed by Sutovsky \& Schatten 1997). Thus, GSH provides the reducing power to initiate chromatin decondensation, prior to the male pronucleus formation (Yoshida et al. 1993, Funahashi et al. 1994). Depletion of endogenous GSH by a specific inhibitor of GSH synthesis during bovine oocyte maturation blocks the formation of a male pronucleus and prevents the assembly of sperm aster microtubules (Sutovsky \& Schatten 1997). The elevated levels of oocyte GSH can enhance male pronuclear formation after IVF (Funahashi et al. 1994). In this study, we found that in comparison to DTT, GSH treatments resulted in much lower values of $\alpha \mathrm{T}$ and COMP $\alpha \mathrm{T}$ in porcine sperm, indicating that GSH treatment does not cause significant DNA denaturation. These results suggested that GSH may function as a disulfide bond reducer with fewer side-effects, thus rendering it more suitable for promoting sperm chromatin decondensation in vitro. In addition, GSH is a potent antioxidant that can counteract the effects of oxidative stress induced sperm DNA damage due to increased reactive oxygen species production and subsequent plasmalemma lipid peroxidation, intracellular enzyme leakage, and eventually chromatin damage (Aitken 1999, Luberda 2001). Thus, the antioxidant property of $\mathrm{GSH}$ may also play a role in protecting porcine sperm
DNA against DTT-induced damage as revealed in our present study.

Furthermore, chromosomal aberrations in ICSI-fertilized embryos were examined to validate the effects of different disulfide bond reducing chemicals on sperm chromatin integrity. We found that treatments with DTT caused more paternal DNA damage with minor or multiple aberrations in porcine sperm than that of GSHtreated and control groups, although the difference did not reach statistical significance. These data were consistent with the results of sperm chromatin structure assay (SCSA). Moreover, these observations favor the idea that in comparison with DTT, GSH is a more suitable agent for promoting porcine sperm chromatin decondensation in porcine ICSI.

We also examined the effects of different disulfide bond reducing chemicals on pronuclear formation in porcine zygotes and the in vitro developmental competence of porcine oocytes fertilized with the treated sperm by ICSI. In comparison with DTT, treatment with GSH resulted in significantly higher rates of zygotes with pronucleus and blastocyst formed. It has been shown that pretreatment of spermatozoa with DTT could enhance the overall success rate of ICSI-fertilized embryos in cows (Rho et al. 1998, Suttern et al. 2000) and minke whales (Asada et al. 2001). By contrast to these previous reports, we did not observe a tendency of increased rate of blastocyst formation in porcine embryos fertilized by ICSI with DTT-treated sperm. However, our data agreed with those of Nakai et al. (2006). The rates of pronucleus and blastocyst formation in vitro could not be improved by spermatozoa treatment with DTT. Ward et al. (2000) found that DTT treatment destabilized sperm nuclear matrix in the presence of an ionic detergent and oocytes injected with these nuclei failed to develop. The discrepancies among these studies may be explained by the fact that although treatment with DTT could reduce $S-S$ in the sperm protamines, simultaneous paternal DNA damage caused by DTT would affect subsequent development of zygotes. In our present study, DTT-treated sperm did not increase the rate of pronuclear and blastocyst formation in comparison with GSH. Intriguingly, we observed a higher haploid rate $(80 \%)$ of the karyotype of ICSI-fertilized blastocysts derived from DTT-treated sperm. The following may offer some hints for the causes of this phenomenon. First, in the majority of ICSI zygotes, the second polar body was extruded by our optimized oocyte activation protocol (Tian et al. 2006) and this may have rendered the zygotes with a haploid suit of maternal chromatin. Alternatively, DTT may elicit harmful effects on porcine sperm chromatin, which in turn causes negative effects on the development of male pronucleus in ICSI-fertilized porcine embryos. Thus, we speculated that the chromatin in haploid ICSI blastocyst was only originated from the maternal chromatin of DTT group. Moreover, our results were similar to those 
reported in humans (Aoki et al. 2005). In human ICSI, it has been shown that the sperm DNA damage is detrimental to fertility and has been regarded as a major factor in lowering embryo quality (Tomsu et al. 2002, Virant-Klun et al. 2002), blastulation rate (Seli et al. 2004), and overall pregnancy rate after IVF (Evenson et al. 2002, Bungum et al. 2004, Henkel et al. 2004, Virro et al. 2004).

Heparin was also used to induce nuclear decondensation (Vendrell et al. 1998, Delgado et al. 2001, Romanato et al. 2003). The glycoprotein heparin receptors are present on human sperm plasma membrane (Delgado et al. 1982). Binding of heparin to its receptors destabilizes sperm plasma membrane that in turn facilitates the incorporation of other molecules such as GSH into sperm nucleus (Marina et al. 2003). Furthermore, the strong binding affinity of heparin for protamines can assist the formation of a highly insoluble complex that may promote sperm chromatin decondensation (reviewed in Romanato et al. 2003). Combination of heparin and DTT may help to induce swelling of sperm nuclear matrix (Jager et al. 1990). These combined actions of both compounds loosen DNA-protamine complexes, which causes a progressive decondensation of nuclear matrix (Leopoldo et al. 2004). Alternatively, the interactions between heparin and GSH may induce sperm nuclear decondensation via an ionic chromatin charge neutralization mechanism (Delgado et al. 2001) in bovine (Delgado et al. 2001), hamster (Francisco et al. 1998), and human (Reyes et al. 2005) zygotes. However, another study found that heparin alone induces sperm nuclear decondensation when only the membranes are severely damaged either by freezing and thawing or by detergents (Jager et al. 1990). In this study, we found that the values of COMP $\alpha \mathrm{T}$ and the parental chromosomal aberrations of DTT-treated porcine sperm did not neither differ significantly from that of DTT + heparin-treated porcine sperm nor between GSH and GSH+heparintreated porcine sperm. Moreover, DTT combined with heparin did not improve the developmental competence of porcine ICSI-fertilized embryos. By contrast, the fertilization rates and developmental competence of porcine ICSI-fertilized embryos derived from GSHtreated spermatozoa were higher than in those derived from GSH+heparin-treated spermatozoa. Why heparin has no detectable effects on porcine sperm chromatin decondensation is not clear. We speculate that the sperm used in our study might be the reason. At the time of freezing, the sperm used were diluted in media with a cryoprotectant. Prior to experimental use, the sperm were only subjected to one cycle of freezing/thawing. Thus, the plasma membrane of the spermatozoa was not destroyed and heparin was unable to enter sperm cells to elicit its effect when used alone. However, we do not know why heparin applied with GSH or DTT did not have synergic effects to induce porcine sperm nuclear decondensation. Whether species differences contribute to the discrepancies of these studies awaits further investigation.

In conclusion, based on our specific oocyte activation protocol, we found that the overall efficiency of porcine ICSI-fertilized embryos was significantly increased when spermatozoa were treated with GSH. Compared with DTT, GSH could decrease DNA breakage in spermatozoa, maintain paternal chromosome integrity, enhance pronuclear formation, and developmental competence of ICSI-fertilized porcine embryos in vitro. Thus, in comparison with the commonly used DTT procedure, our present study demonstrated that GSH is a more suitable disulfide bond reducing agent for preparing porcine sperm for ICSI.

\section{Materials and Methods Chemicals and reagents}

All chemicals and reagents, unless otherwise stated, were purchased from Sigma-Aldrich Co. Porcine follicular fluid (pFF) was aspirated from 3-6 mm diameter follicles from ovaries collected at a local slaughterhouse (Beijing Jingbei Slaughterhouse, Beijing, China). The follicular fluids were collected under sterile conditions and stored at $-20^{\circ} \mathrm{C}$. Prior to use, the follicular fluids were centrifuged at $1500 \mathrm{~g}$ for $30 \mathrm{~min}\left(4^{\circ} \mathrm{C}\right)$ as described previously (Abeydeera et al. 1998).

\section{Sperm preparation}

Fresh semen was diluted 1:10 with the TNE buffer (0.01 M Tris, $0.15 \mathrm{M} \mathrm{NaCl}, 1 \mathrm{mM}$ EDTA, pH 7.4) supplemented with $10 \%$ $(\mathrm{v} / \mathrm{v})$ glycerol, and then frozen and stored in liquid nitrogen until in use. Frozen semen samples as described above were thawed at $37^{\circ} \mathrm{C}$. Spermatozoa were collected by centrifugation $(600 \mathrm{~g}, 6 \mathrm{~min})$ and resuspended in TNE buffer at a concentration of $1 \times 10^{6} / \mathrm{ml}$. The sperm solution was allocated for treatment with either GSH $(8 \mathrm{mM}, 120 \mathrm{~min})$, DTT $(5 \mathrm{mM}$, $60 \mathrm{~min}), \mathrm{GSH}(8 \mathrm{mM})$, and heparin $(10 \mu \mathrm{g} / \mathrm{ml})$ for $60 \mathrm{~min}$, or DTT $(5 \mathrm{mM})$ and heparin $(10 \mu \mathrm{g} / \mathrm{ml})$ for $60 \mathrm{~min}$ at $39^{\circ} \mathrm{C}$. Sperm without any chemical pretreatment served as the control group. After incubation, the sperm samples were washed three times with TNE buffer by centrifugation ( $600 \mathrm{~g}$, $6 \mathrm{~min}$ ) to remove the chemicals. After the last wash, the sperm pellet was resuspended in TNE buffer and kept on ice until use.

In this study, the treatment time and concentrations of DTT and heparin were chosen based on previous literature (Rho et al. 1998, Delgado et al. 2001) and the treatment time and concentrations of GSH were selected from our pre-experiments (data not shown) by the higher rate of sperm nuclear swelling under phase contrast light microscopy.

\section{AO staining and flow cytometry}

The chromatin integrity of spermatozoa was examined by $\mathrm{AO}$ staining and flow cytometry as previously described (Evenson et al. 1986, Evenson 1990). Semen samples $(200 \mu \mathrm{l})$ were treated with $400 \mu \mathrm{l}$ of a low $\mathrm{pH}$ solution with detergent $(0.1 \%$ 
Triton $\mathrm{X}-100(\mathrm{v} / \mathrm{v}), 0.15 \mathrm{M} \mathrm{NaCl}$ and $0.08 \mathrm{M} \mathrm{HCl}, \mathrm{pH} 1.2)$ for $30 \mathrm{~s}$. Triton X-100 treatment permeabilizes sperm cell membranes and thus provides greater accessibility of $\mathrm{AO}$ to stain DNA. After the $30 \mathrm{~s}$ acid treatment, $1.2 \mathrm{ml} \mathrm{AO}$ staining buffer $\left(6 \mu \mathrm{g} / \mathrm{ml} \mathrm{AO}, 0.1 \mathrm{M}\right.$ citric acid, $0.2 \mathrm{M} \mathrm{Na}_{2} \mathrm{HPO}_{4}, 1 \mathrm{mM}$ EDTA, $0.15 \mathrm{M} \mathrm{NaCl}, \mathrm{pH}$ 6.0) were added to stain DNA for $3 \mathrm{~min}$. The spermatozoa were then analyzed by a FACS Calibur flow cytometer (Becton Dickinson, San Josè, CA, USA), equipped with a $6 \mathrm{~W}$ Argon ion laser (Innova 306, Coherent, Santa Clara, CA, USA), tuned at $488 \mathrm{~nm}$ and operated at a power output of $300 \mathrm{~mW}$, light mode. Green $(530 \pm 30 \mathrm{~nm})$ and red $(>630 \mathrm{~nm})$ fluorescence intensities, corresponding to amounts of native and denatured DNA respectively, were recorded from $1 \times 10^{4}$ spermatozoa per sample. All measurements were initiated at $3 \mathrm{~min}$ after $\mathrm{AO}$ staining with a flow rate of $\sim 200$ cells/s. Scattergram analysis was carried out using the Cell Quest Pro version 4.0 software (Becton Dickinson Biosciences). Each data point represents the coordinate of red and green fluorescence intensities for an individual spermatozoon. Events accumulated in the lower left corner correspond to cell debris and were excluded from the analysis (Fig. 1). The bivariate data can be conveniently expressed by the function $\alpha \mathrm{T}$, which is the ratio of red to total (red plus green) fluorescence intensity (Darzynckiewicz et al. 1975), thus representing the amount of denatured, single-stranded DNA over total cellular DNA. $\alpha$ T was calculated (ListView; Phoenix Flow Systems, San Diego, CA, USA) for each sample. The results were expressed as the mean $(\mathrm{X} \alpha \mathrm{T})$ and the $\mathrm{SD}(\mathrm{SD} \alpha \mathrm{T})$ of $\alpha \mathrm{T}$ distribution, and the frequency of cells with high $\alpha \mathrm{T}$ values referred as to COMP $\alpha \mathrm{T}$. The values of $\alpha \mathrm{T}$ ranged between 0 and 1, but were converted to 0-1000 numerical scale to facilitate working with integral numbers. Additional parameter in this flow cytometry based sperm chromatin structure assay included the mean ( $X$ Green) of the green fluorescence intensity.

\section{Oocyte collection and in vitro maturation (IVM)}

Porcine ovaries were obtained from gilts at a local abattoir and transported to the laboratory in sterile physiological saline (Beijing Chemical Reagent Co., Beijing, China) containing $75 \mathrm{mg} / \mathrm{l}$ penicillin (Huabei Medicine Co. Ltd, Shijiazhuang, China) and $50 \mathrm{mg} / \mathrm{l}$ streptomycin (Hebei Yuanzheng Medicine Co. Ltd, Shijiazhuang, China) at $30{ }^{\circ} \mathrm{C}$ to $35^{\circ} \mathrm{C}$ within $2 \mathrm{~h}$. The ovaries were washed three times with fresh saline and stored in saline at $37^{\circ} \mathrm{C}$. The cumulus-oocyte complexes (COCs) were aspirated from the antral follicles (3-6 $\mathrm{mm}$ in diameter) using a 16 -gauge needle attached to a $10 \mathrm{ml}$ disposable syringe. The aspirates were transferred into a $15 \mathrm{ml}$ plastic centrifuge tube pre-warmed to $37^{\circ} \mathrm{C}$ in a water bath. The sediments were washed twice with HEPES-buffered Tyrode albumin lactate pyruvate medium (TL-HEPES; Bavister et al. 1983) containing $0.3 \%$ (w/v) BSA (Roche Diagnostics Corporation). Only those oocytes surrounded by at least three layers of compact cumulus cells and with uniform cytoplasm were selected for experimental use. The COCs were washed three times with fresh maturation medium composed of TCM199 medium (Earle's Salts; GiBco, Life Technologies Inc.; Abeydeera et al. 2000, Hyun et al. 2003) supplemented with $10 \%(\mathrm{v} / \mathrm{v}) \mathrm{pFF}, 0.1 \mathrm{mg} / \mathrm{ml}$ cysteine, $10 \mathrm{ng} / \mathrm{ml}$ epidermal growth factor, $10 \mathrm{IU} / \mathrm{ml}$ eCG (Intervet Pty. Ltd, Boxmeer, Australia), $10 \mathrm{IU} / \mathrm{ml}$ hCG (Intervet Pty. Ltd), $0.065 \mathrm{mg} / \mathrm{ml}$ penicillin and $0.05 \mathrm{mg} / \mathrm{ml}$ streptomycin. Selected COCs (25-30/group) were transferred to a droplet of $100 \mu \mathrm{l}$ maturation medium covered with mineral oil in a petri dish (Nunc, Nalge Nunc International, Roskilde, Denmark). IVM was carried out at $39{ }^{\circ} \mathrm{C}$ in an atmosphere of $5 \% \mathrm{CO}_{2}$ in humidity air for $44 \mathrm{~h}$ as described previously (Abeydeera et al. 2001).

\section{ICSI}

After IVM, COCs with expanded layers of cumulus cells were treated with $0.1 \%(\mathrm{w} / \mathrm{v})$ hyaluronidase. Cumulus cells were then removed by repeated gentle pipetting. Denuded oocytes were placed in droplets of TCM199-HEPES containing $0.5 \%$ $(\mathrm{v} / \mathrm{v})$ fetal bovine serum (FBS; Hyclone, Logan, Utah, USA). Denuded oocytes with uniform cytoplasm were chosen for the following experiments.

ICSI was performed by using an inverted microscope (Olympus IX70, Olympus optical Co. Ltd., Tokyo, Japan) with micromanipulators (Eppendorf, Hamburg, Germany). To accommodate the bigger size of a porcine spermatozoon, the injection pipettes had to be $6-7 \mu \mathrm{m}$ in their inner diameter. A $100 \mu \mathrm{l}$ drop of HEPES-M199 containing 0.5\% (v/v) FBS and a $20 \mu \mathrm{l}$ drop of $10 \%(\mathrm{w} / \mathrm{v})$ polyvinylpyrrolidone (PVP) was placed in the center of a $35 \mathrm{~mm}$ dish covered with mineral oil. For each group of injections, 20-30 oocytes were placed in a $100 \mu \mathrm{l}$ drop of medium and the $20 \mu \mathrm{l}$ sperm suspension was transferred to the PVP drop that had been preequilibrated for $2 \mathrm{~h}$. The oocyte was held with a holding pipette in an orientation so that the first polar body was at the 6 or 12 o'clock position. A spermatozoon was then injected into the cytoplasm with a micropipette.

\section{Activation of oocytes following ICSI}

After spermatozoon injection, oocytes were transferred into an electrical activation medium (0.3 M mannitol, $0.05 \mathrm{mM} \mathrm{CaCl}_{2}$, $0.1 \mathrm{mM} \mathrm{MgCl}_{2} ;$ Zimmermann \& Viemken 1982). The oocytes were then placed in a chamber connected to an electrical pulse generator (Fujira Industry Co. Ltd, Tokyo, Japan) with two parallel electrodes at a distance of $0.46 \mathrm{~mm}$. Direct current pulse $(1.3 \mathrm{kV} / \mathrm{cm}, 1 \times 80 \mu \mathrm{s})$ was applied to activate the oocytes (Zhu et al. 2002). After electrical activation, the oocytes were washed three times with PZM-3 (Yoshioka et al. 2002) medium containing $3 \mathrm{mg} / \mathrm{ml}$ BSA and cultured in the same medium for $3 \mathrm{~h}$. The ICSI oocytes were then cultured for another $6 \mathrm{~h}$ in droplets of PZM-3 medium containing $3 \mathrm{mg} / \mathrm{ml}$ BSA supplemented with $2 \mathrm{mM}$ 6-DMAP for chemical activation.

\section{In vitro embryo culture}

The ICSI oocytes were washed three times with PZM-3 medium containing $3 \mathrm{mg} / \mathrm{ml}$ BSA. 15-20 oocytes/groups were cultured in a $50 \mu \mathrm{l}$ droplet of PZM-3 medium droplets containing $3 \mathrm{mg} / \mathrm{ml}$ BSA under mineral oil at $39{ }^{\circ} \mathrm{C}$ in a humidified air of $5 \% \mathrm{CO}_{2}$ for 7 days. The cleavage and blastocyst rates of the ICSI 
oocytes were recorded at day 2 and 7 respectively. Three replicates were performed for this experiment.

\section{Chromosomal analysis}

Following ICSI and electrical activation, the zygotes were cultured in droplets of PZM-3 medium containing $3 \mathrm{mg} / \mathrm{ml}$ BSA for $6 \mathrm{~h}$ and then transferred into PZM-3 medium containing $3 \mathrm{mg} / \mathrm{ml}$ BSA supplemented with $0.05 \mu \mathrm{g} / \mathrm{ml}$ of colchicine. Between 19 and $21 \mathrm{~h}$ after ICSI, eggs arrested at the metaphase of the first cleavage were treated with $1 \%$ pronase (1000 tyrosine units/mg, Sigma) for $5 \mathrm{~min}$ at room temperature to soften the zonae pellucidae. The zygotes were then treated with a hypotonic solution composed of a mixture of $1 \%$ sodium citrate and $30 \%$ FBS $(1: 1, v / v)$ for $5 \mathrm{~min}$ at $37^{\circ} \mathrm{C}$ or $10 \mathrm{~min}$ at room temperature. Chromosomes were spread on slides by the gradual fixation/air-drying method as described (Mikamo \& Kamiguchi 1983). Briefly, zygotes with a small amount of hypotonic solution were transferred into fixative I (methanol:glacial acetic acid: $\mathrm{H}_{2} \mathrm{O}$ $=5: 1: 4)$ for $3-5 \mathrm{~min}$ until they turned pale. The zygotes were then aspirated into a fine-mouthed glass pipette and released within a circle that had been made on the reverse side of a grease-free glass slide. The zygotes were covered immediately with fixative II (methanol:glacial acetic acid=3:1) released from another pipette. The slides were kept in a jar filled with fixative II for $5 \mathrm{~min}$. Finally, the slides were placed into fixative II (methanol:glacial acetic acid: $\mathrm{H}_{2} \mathrm{O}=3: 3: 1$ ) for $1 \mathrm{~min}$, removed slowly and dried in a stream of warm moist air (Rosenbusch et al. 1998). The samples were stained with $10 \mu \mathrm{g} / \mathrm{ml}$ Hoechst 33342 (Sigma) for $10 \mathrm{~min}$ and observed at $\times 1000$ magnification with oil-immersion optics using u.v. illumination for conventional chromosome analysis under a microscope (Olympus BX60, Olympus Optical Co. Ltd). A spermatozoon was classified to be chromosomally normal when a zygote contained 38 structurally normal chromosomes (19 pairs) at metaphase of the first mitotic division. We looked at the percentage of normal and abnormal metaphases (differentiating between metaphases with minor and multiple aberrations). When more than nine aberrations per karyoplate were observed, we categorized them as multiple aberrations. The number of aberrations per spermatozoon, which was calculated by dividing the total number of aberrations by the total number of karyoplates examined in one treatment, represented the intensity of the chromosomal damage. Four replicates were performed of this experiment (Szczygiel \& Ward 2002).

\section{Fixation and staining of zygotes}

At $15 \mathrm{~h}$ after ICSI, zygotes cultured in PZM-3 medium were fixed and stained to assess pronuclear formation as follows. The zygotes were mounted on glass slides, fixed in acetic acid:alcohol $(1: 3, v / v)$ for $48 \mathrm{~h}$, stained with $1 \%(\mathrm{w} / \mathrm{v})$ orcein in $45 \%(\mathrm{v} / \mathrm{v})$ acetic acid in water. Pronuclear formation was examined under a phase contrast microscope (Olympus IX70, Olympus Optical Co. Ltd). Oocytes with one pronucleus and a non-condensing sperm head were classified as activated, whereas those with two pronuclei and without a noncondensing sperm head were regarded as fertilized (Rho et al. 1998, Lee et al. 2003).

\section{Chromosome analysis of ICSI blastocysts}

Blastocysts were synchronized at metaphase by the addition of $0.5 \mu \mathrm{g} / \mathrm{ml}$ colchicine to the culture medium for $4 \mathrm{~h}$, followed by incubation in $1 \%(\mathrm{w} / \mathrm{v})$ sodium citrate for $20 \mathrm{~min}$ at $4{ }^{\circ} \mathrm{C}$. Each individual blastocyst in $1.5 \mu \mathrm{l}$ methanol:acetic acid $(1: 1)$ was placed on a pre-cleaned microscope slide and blown upon to spread the chromosomes as they dried. The samples were stained in Hoechst $33342(10 \mu \mathrm{g} / \mathrm{ml})$ and chromosomes were examined under u.v. illumination under a microscope as described above. Blastocysts were classified as diploid (2n), haploid ( $1 \mathrm{n}$ ), and polyploid/mixoploid ( $\geq 3 \mathrm{n} /$ blastocysts with blastomeres of different ploidy) according to the chromosome numbers.

\section{Statistical analysis}

Analysis of variance was carried out by use of ANOVA (PROC GLM) using the SAS statistical package (SAS Institute Inc., Cary, NC, USA). All percentage data were subjected to arcsine transformation before statistical analysis. Duncan's multiple range tests and $\chi^{2}$ were used to compare values of each individual treatment. $P<0.05$ was considered to be statistically significant.

\section{Declaration of interest}

The authors declare that there is no conflict of interest that could be perceived as prejudicing the impartiality of the research reported.

\section{Funding}

The present study was partially supported by a grant (\#30671507) from the National Natural Science Foundation of China and a grant (\#2006BAD14B08) from the National key technology R\&D program.

\section{Acknowledgements}

We thank the Beijing Hao-bang Pig Artificial Insemination Service Co., Ltd. for supplying fresh boar semen and the Beijing Jingbei Slaughterhouse for supplying porcine ovaries. We also thank Mr Yongqiang Wang, College of Veterinary Medicine, China Agricultural University, for his technical assistance.

\section{References}

Abeydeera LR, Wang WH, Cantley TC, Prather RS \& Day BN 1998 Presence of $\beta$-mercaptoethanol can increase the glutathione content of pig oocytes matured in vitro and the rate of blastocyst development after in vitro fertilization. Theriogenology 50 747-756. 
Abeydeera LR, Wang WH, Cantley TC, Rieke A, Murphy CN, Prather RS \& Day BN 2000 Development and viability of pig oocytes matured in a protein-free medium containing epidermal growth factor. Theriogenology 54 787-797.

Abeydeera LR, Wang WH, Prather RS \& Day BN 2001 Effect of incubation temperature on in vitro maturation of porcine oocytes: nuclear maturation, fertilization and developmental competence. Zygote $\mathbf{9}$ 331-337.

Aitken RJ 1999 The Amoroso lecture. The human spermatozoon - a cell in crisis? Journal of Reproduction and Fertility 115 1-7.

Aoki VW, Moskovtsev SI, Willis J, Liu L, Mullen JBM \& Carrell DT 2005 DNA integrity is compromised in protamine-deficient human sperm. Journal of Andrology 26 741-748.

Asada M, Wei H, Nagayama R, Tetsuka M, Ishikawa H, Ohsumi S \& Fukui Y 2001 An attempt at intracytoplasmic sperm injection of frozen-thawed minke whale (Balaenoptera bonaerensis) oocytes. Zygote 9 299-307.

Bavister BD, Leibfried ML \& Liebermann G 1983 Development of preimplantation embryos of the golden hamster in a defined culture medium. Biology of Reproduction 28 235-247.

Bungum M, Humaidan P, Spano M, Jepson K, Bungum L \& Giwercman A 2004 The predictive value of sperm chromatin structure assay (SCSA) parameters for the outcome of intrauterine insemination, IVF and ICSI. Human Reproduction 19 1401-1408.

Darzynckiewicz Z, Traganos F, Sharpless T \& Melamed MR 1975 Thermal denaturation of DNA in situ as studied by acridine orange staining and automated cytofluorometry. Experimental Cell Research 90 411-428.

Delgado N, Reyes R, Huacuja L, Merchant H \& Rosado A 1982 Heparin binding sites in the human spermatozoa membrane. Archives of Andrology 8 87-95.

Delgado NM, Flores-Alonso JC, Rodriguez-Hernandez HM, MerchantLarios H \& Reyes R 2001 Heparin and glutathione II: correlation between decondensation of bull sperm cells and its nucleons. Archives of Andrology 47 47-58.

Evenson DP 1990 Flow cytometric analysis of male germ cell quality. Methods in Cell Biology 33 401-410.

Evenson DP, Darzynkiewicz Z \& Melamed MR 1980 Relation of mammalian sperm chromatin heterogeneity to fertility. Science $2101131-1133$.

Evenson DP, Darzynkiewicz Z \& Jost LK 1986 Changes in accessibility of DNA to various fluorochromes during spermatogenesis. Cytometry $745-53$.

Evenson DP, Jost LK, Baer RK, Turner TW \& Schrader SM 1991 Individuality of DNA denaturation patterns in human sperm as measured by the sperm chromatin structure assay. Reproductive Toxicology 5 115-125.

Evenson DP, Larson KL \& Jost LK 2002 Sperm chromatin structure assay: its clinical use for detecting sperm DNA fragmentation in male infertility and comparisons with other techniques. Journal of Andrology 23 25-43.

Francisco JV, Carmen R \& Juan JT 1998 The heparin-glutathione test: an alternative to the hypo-osmotic swelling test to select viable sperm for intracytoplasmic sperm injection. Fertility and Sterility 70 1156-1161.

Funahashi H, Cantley TC, Stumpf TT, Terlouw SL \& Day B 1994 Use of lowsalt culture medium with elevated oocyte glutathione levels and enhanced male pronuclear formation after in vitro fertilization. Biology of Reproduction $\mathbf{5 1}$ 633-639.

Henkel R, Hajimohammad M, Stalf T, Hoogendijk C, Mehnert C, Menkveld R, Gips H, Schill WB \& Kruger TF 2004 Influence of deoxyribonucleic acid damage on fertilization and pregnancy. Fertility and Sterility 81 965-972.

Hyun SH, Lee GS, Kim DY, Kim HS, Lee SH, Kim S, Lee ES, Lim JM, Kang SK, Lee BC \& Hwang WS 2003 Effect of maturation media and oocytes derived from sows or gilts on the development of cloned pig embryos. Theriogenology 59 1641-1649.

Jager S, Wijchman J \& Kremer J 1990 Studies on the decondensation of human, mouse, and bull sperm nuclei by heparin and other polyanions. Journal of Experimental Zoology 256 315-322.

Kaneko T, Whittingham DG, Overstreet JW \& Yanagimachi R 2003 Tolerance of the mouse sperm nuclei to freeze-drying depends on their disulfide status. Biology of Reproduction 69 1859-1862.

Katayama M, Rieke A, Cantley T, Murphy C, Dowell L, Sutovsky P \& Day BN 2007 Improved fertilization and embryo development resulting in birth of live piglets after intracytoplasmic sperm injection and in vitro culture in a cysteine-supplemented medium. Theriogenology 67 835-847.

Kolbe T \& Holtz W 1999 Intracytoplasmic injection (ICSI) of in vivo or in vitro matured oocytes with fresh ejaculated or frozen-thawed epididymal spermatozoa and additional calcium-ionophore activation in the pig. Theriogenology 52 671-682.

Kolbe T \& Holtz W 2000 Birth of piglet derived from an oocyte fertilized by intracytoplasmic sperm injection (ICSI). Animal Reproduction Science 64 97-101.

Kren R, Kikuchi K, Nakai M, Miyano T, Ogushi S, Nagai T, Suzuki S \& Fulka J 2003 Intracytoplasmic sperm injection the pig: where is the problem? Journal of Reproduction and Development 49 271-273.

Lai LX, Sun QY, Wu GM, Murphy CN, Kühholzer B, Park KW, Bonk AJ, Day BN \& Prather RS 2001 Development of porcine embryos and offspring after intracytoplasmic sperm injection with liposome transfected or non-transfected sperm into in vitro matured oocytes. Zygote 9 339-346.

Lalich RA, Vedantham S, McCormick N, Wagner C \& Prins GS 1989 Relationship between heparin binding characteristics and ability of human spermatozoa to penetrate hamster ova. Journal of Reproduction and Fertility 86 297-302.

Lee JW, T XC \& Yang XZ 2003 Failure of male pronucleus formation is the major cause of lack of fertilization and embryo development in pig oocytes subjected to intracytoplasmic sperm injection. Biology of Reproduction 68 1341-1347.

Leopoldo S, Alberto Mantovani M \& Simonetta P 2004 The partial head decondensation test is a new, quick method to assess acrosome status in human spermatozoa. Fertility and Sterility 81 1007-1012.

Luberda Z 2001 Współczesne poględy dotyczęce wpływu reaktywnych form tlenu na funkcje plemników u ssaków (Present conception regarding the effect on reactive oxygen species on functions of mammalian spermatozoa). In Polish with English summary. Postępy Biologii Komórki 28 309-316.

Marina R, Mónica SC, Gabriel B, Consuelo B, Juan CC \& Lucrecia C 2003 Heparan sulphate: a putative decondensing agent for human spermatozoa in vivo. Human Reproduction 18 1868-1873.

Martin MJ 2000 Development of in vivo-matured porcine oocytes following intracytoplasmic sperm injection. Biology of Reproduction 63 109-112.

Mikamo K \& Kamiguchi Y 1983 A New Assessment System for Chromosomal Mutagenicity Using Oocytes and Early Zygotes of the Chinese Hamster. New York: Alan R.

Montag M, Tok V, Liow S, Bongson A \& Nicolle JC 1992 In vitro decondensation of mammalian sperm and subsequent formation of pronuclei-like structures for micromanipulation. Molecular Reproduction and Development 33 338-346.

Nakai M, Kashiwazaski N, Takizawa A, Hayashi Y, Nakatsukasa E, Fuchimoto D, Noguchi J, Kaneko H, Shino M \& Kikuchi K 2003 Viable piglets generated from porcine oocytes matured in vitro and fertilized by intracytoplasmic sperm injection. Biology of Reproduction $\mathbf{6 8}$ 1003-1008.

Nakai M, Kashiwazaki N, Takizawa A, Maedomari N, Ozawa M, Noguchi J, Kaneko H, Shino M \& Kikuchi K 2006 Morphologic changes in boar sperm nuclei with reduced disulfide bonds in electrostimulated porcine oocytes. Reproduction 131 603-611.

Pérez-Crespo M, Moreira P, Pintado B \& Gutiérrez-Adán A 2008 Factors from damaged sperm affect its DNA integrity and its ability to promote embryo implantation in mice. Journal of Andrology 29 47-54.

Perreault SD, Wolff RA \& Zirkin BR 1984 The role of disulfide bond reduction during mammalian sperm nuclear decondensation in vitro. Developmental Biology 101 160-167.

Perreault SD, Barbee RR \& Slott VL 1988 Importance of glutathione in the acquisition and maintenance of sperm nuclear decondensing activity in maturing hamster oocytes. Developmental Biology 125 181-186.

Perry ACF, Wakayama T, Kishikawa H, Kasai T, Okabe M, Toyoda Y \& Yanagimachi R 1999 Mammalian transgenesis by intracytoplasmic sperm injection. Science 284 1180-1183.

Reyes R, Rosado A, Hernández O \& Delgado NM 2005 Heparin and glutathione: physiological decondensing agents of human sperm nuclei. Gamete Research 23 39-47.

Rho GJ, Kawarsky S, Johuson WH, Kochhar K \& Betteridge KJ 1998 Sperm and oocyte treatments to improve the formation of male and female 
pronuclei and subsequent development following intracytoplasmic sperm injection into bovine oocytes. Biology of Reproduction 59 918-924.

Romanato M, Cameo MS, Bertolesi G, Baldini C, Calvo JC \& Calvo L 2003 Heparin sulphate: a putative decondensing agent for haman spermatozoa in vivo. Human Reproduction 19 1868-1873.

Rosenbusch B, Schneider M \& Sterzik K 1998 Chromosomal analysis of multipronuclear zygotes obtained after partial zona dissection of the oocytes. Molecular Human Reproduction 4 1065-1070.

Seli E, Gardner DK, Schoolcraft WB, Moffatt O \& Sakkas D 2004 Extent of nuclear DNA damage in ejaculated spermatozoa impacts on blastocyst development after in vitro fertilization. Fertility and Sterility 82 378-383.

Spanò M \& Evenson DP 1993 Flow cytometric analysis for reproductive biology. Biology of the Cell 78 53-62.

Sutovsky P \& Schatten G 1997 Depletion of glutathione during bovine oocyte maturation reversibly blocks the decondensation of the male pronucleus and pronuclear apposition during fertilization. Biology of Reproduction 56 1503-1512.

Sutovsky P, Tengowski MW, Navara CS, Zoran SS \& Schatten G 1997 Mitochondrial Sheath Movement and Detachment in Mammalian. But Not Nonmammalian, Sperm Induced by Disulfde Bond Reduction. Molecular Reproduction and Development 47 79-86.

Suttern R, Zakhartchenko V, Stojkovic P, Muller S, Alberio R, Medjugorac I, Brem G, Wolf E \& Stojkovic M 2000 Intracytoplasmic sperm injection in bovine: effects of oocyte activation, sperm pretreatment and injection technique. Theriogenology 54 935-948.

Szczygiel MA \& Ward WS 2002 Combination of dithiothreitol and detergent treatment of spermatozoa causes paternal chromosomal damage. Biology of Reproduction 67 1532-1537.

Tateno H \& Kamiguchi Y 1999 Dithiothreitol induces sperm nuclear decondensation and protects against chromosome damage during male pronuclear formation in hybrid zygotes between Chinese hamster spermatozoa and Syrian hamster oocytes. Zygote 7 321-327.

Tesarik J 1996 Fertilization of oocytes by injecting spermatozoa, spermatids and spermatocytes. Reviews of Reproduction 1 149-152.

Thibault C 1972 Final stages of oocyte maturation. In Oogenesis, pp 397-411. Eds JD Biggers \& AW Schuetz. Baltimore: University Park Press.

Tian JH, Wu ZH, Liu L, Cai Y, Zeng SM, Zhu SE, Liu GS, Li Y \& Wu CX 2006 Effects of oocyte activation and sperm preparation on the development of porcine embryos derived from in vitro matured oocytes and intracytoplasmic sperm injection. Theriogenology 66 439-448.

Tomsu M, Sharma V \& Miller D 2002 Embryo quality and IVF treatment outcomes may correlate with different sperm comet assay parameters. Human Reproduction 17 1856-1862.

Vendrell FJ, Rubio C \& Tarín JJ 1998 The heparin-glutathione test: an alternative to the hypo-osmotic swelling test to select viable sperm for intracytoplasmic sperm injection. Fertility and Sterility 70 1156-1161.
Virant-Klun I, Tomazevic T \& Meden-Vrtovec H 2002 Sperm singlestranded DNA, detected by acridine orange staining, reduces fertilization and quality of ICSI-derived embryos. Journal of Assisted Reproduction and Genetics 19 319-328.

Virro MR, Larson-Cook KL \& Evenson DP 2004 Sperm chromatin structure assay (SCSA) parameters are related to fertilization, blastocyst development, and ongoing pregnancy in in vitro fertilization and intracytoplasmic sperm injection cycles. Fertility and Sterility 81 1289-1295.

Ward MA \& Ward WS 2004 A model for the function of sperm DNA degradation. Reproduction, Fertility and Development $\mathbf{1 6}$ 547-554.

Ward WS, Kishikawa H, Akutsu H, Yanagimachi H \& Yanagimachi R 2000 Further evidence that sperm nuclear proteins are necessary for embryogenesis. Zygote 8 51-56.

Watanabe H \& Fukui Y 2006 Effects of dithiothreitol and boar on pronuclear formation and embryonic development following intracytoplasmic sperm injection in pigs. Theriogenology 65 528-539.

Yamauchi Y, Ajduk A, Riel JM \& Ward MA 2007 Ejaculated and epididymal mouse spermatozoa are different in their susceptibility to nucleasedependent DNA damage and in their nuclease activity. Biology of Reproduction 77 636-647.

Yong HY, Hong JY, Kang SK, Lee BC, Lee ES \& Hwang WS 2005 Sperm movement in the ooplasm, dithiothreitol pretreatment and sperm freezing are not required for the development of porcine embryos derived from injection of head membrane-damaged sperm. Theriogenology 63 783-794.

Yoshida M, Ishigaki K, Nagai T, Chikyu M \& Pursel VG 1993 Glutathione concentration during maturation and after fertilization in pig oocytes: relevance to the ability of oocytes to form male pronucleus. Biology of Reproduction 49 89-94.

Yoshioka K, Suzuki T, Tanaka A, Anas IMK \& Iwamura S 2002 Birth of piglets derived from porcine zygotes cultured in a chemically defined medium. Biology of Reproduction 66 112-119.

Zhu J, Evelyn ET, Judy F, Anthea S, John RD, Paul ADS \& Ian W 2002 Improvement of an electrical activation protocol for porcine oocytes. Biology of Reproduction 66 635-641.

Zimmermann U \& Viemken J 1982 Electric field-induced cell-to-cell fusion. Journal of Membrane Biology 67 165-182.

Zirkin BR, Perreault SD \& Naish SJ 1989 Formation and function of the male pronucleus during mammalian fertilization. In The Molecular Biology of Fertilization, pp 91-114. San Diego, CA: Academic Press.

Received 31 March 2008

First decision 23 April 2008

Revised manuscript received 11 December 2008

Accepted 20 January 2009 